\title{
Control de la chinche de los pastos Collaria scenica (Hemiptera: Miridae) con nematodos entomopatógenos en invernadero
}

\author{
Natalia Naranjo ${ }^{1}$, Daniel A. V. Montero² \& Adriana Sáenz ${ }^{3}$ \\ ${ }^{1}$ Universidade Estadual Paulista Júlio de Mesquita Filho, Faculdade de Ciências Agrárias e Veterinárias de Jaboticabal, Departamento de Fitossanidade, Via de Acesso Prof . Paulo \\ Donato Castelane, Rural, CEP 14884-900, Jaboticabal-SP, Brasil. E-mail: nnaranjoguevara@gmail.com \\ ${ }^{2}$ Universidade Estadual Paulista Júlio de Mesquita Filho, Faculdade de Ciências Agronômicas de Botucatu, Departamento de Horticultura, Laboratório de Plantas Medicinais, Rua \\ Jose Barbosa de Barros, 1780, Parque dos Pinheiros, CEP 18610-307, Botucatu-SP, Brasil. Caixa Postal 237. E-mail:dvillamontero@fca.unesp.br; \\ ${ }_{3}^{3}$ Pontificia Universidad Javeriana, Facultad de Ciencias, Departamento de Biología, Unidad de Ecología y Sistemática, Laboratorio de Control Biológico, Carrera 7 No 43-82, \\ Edificio 54, Oficina 200, Bogotá D.C., Colombia. E-mail: adriana.saenz@javeriana.edu.co
}

\section{RESUMEN}

Se estudió la eficacia de juveniles infectivos (JI) de Steinernema sp. JCL024 y Heterorhabditis sp. SL0708 sobre adultos y ninfas de Collaria scenica Stal bajo condiciones de invernadero. Se evaluaron 5 dosis (2, 16, 78, $160 \mathrm{Jls}) / \mathrm{cm}^{2}$ foliolo) para cada nematodo. El experimento fue observado durante 21 días cada 24 horas, realizando el conteo de mortalidad y valoración del daño del pasto kikuyo. Las chinches presentaron $100 \%$ de mortalidad después de 15 días. Cada chinche produjo en promedio $13.000 \mathrm{JI} / 6$ días. En cuanto al daño, no alcanzó el nivel 2 en los 21 días respecto al tratamiento sin nematodos que presentó daño 3 con necrosis y enrollamiento apical de las hojas. Por ende, Steinernema sp. JCL024 y Heterorhabditis sp. SL0708 ejercieron control en la chinche de los pastos y mantuvieron bajo el daño en el follaje.

Palabras clave: control biológico, juvenil infectivo, Steinernema sp., Heterorhabditis sp., Pennisetum clandestinum

\section{Control of pasture Collaria scenica bug (Hemiptera: Miridae) with entomopathogenic nematodes in greenhouse}

\begin{abstract}
We studied the efficacy of infective juveniles (IJ) of Steinernema sp. strain JCL024 and Heterorhabditis sp. strain SL0708 on adults and nymphs of Collaria scenica under greenhouse conditions. Five doses were evaluated $(2,16,78,160 \mathrm{lJ}) \mathrm{cm}^{-2}$ leaf) for each nematode. The experiment was evaluated for 21 days every 24 hours, making mortality counts and assessment of damage on Kikuyo grass. The bugs showed $100 \%$ mortality after 15 days. Each bug produced an average of $13,000 \mathrm{IJ} / 6$ days. As for the damage did not reach level 2 within 21 days prior to treatment without nematodes that damage introduced 3 with necrosis and apical leaf curl. Thus, Steinernema sp. strain JCL024 and Heterorhabditis sp. strain SL0708 exercised control bug on the grass and kept under the level of damage to the foliage.
\end{abstract}

Key words: biological control, infective juvenile, Steinernema sp., Heterorhabditis sp., Pennisetum clandestinum 


\section{INTRODUCCIÓN}

La chinche de los pastos Collaria scenica Stal, es un hemíptero de la familia Miridae que se encuentra en gramíneas cultivadas y no cultivadas (Martínez \&Barreto, 1998). Presenta varios hospederos alternativos lo que facilita su reproducción y sobrevivencia durante todo el año. Este insecto chupador genera un daño directo al follaje causado por la inserción del aparato bucal en las nervaduras y la ingestión del contenido celular, provocando manchas cloróticas que disminuyen el área fotosintética de las plantas (Estrada, 2002; Ferreira et al., 2001) y reducción hasta un $25 \%$ de la disponibilidad de biomasa forrajera y de ingresos económicos (Vergara, 2006).

La chinche de los pastos ha sido reportada en varias regiones de Argentina, Uruguay, Colombia, Venezuela y Brasil (Briceño, 2007). En este último, según Carlessi et al. (1999), ha llamado la atención la intensidad con la que se ha presentado en trigo (Triticum aestivum L). Otras especies del género Collaria tienen amplia distribución en el continente americano y son consideradas plagas de importancia económica en algunas gramíneas especialmente en forrajeras (Melo et al., 2004).

En Colombia, C. scenica se ha convertido en la mayor plaga limitante para el desarrollo de la ganadería lechera, debido a que ataca agresivamente a Pennisetum clandestinum (HochstChiov) conocido como pasto kikuyo (Barboza, 2009; Vergara, 2006). Esta especie de pasto importada de África Oriental es la principal fuente de forraje que conforma los potreros en las zonas de ganadería lechera en Colombia y Venezuela (Briceño, 2007; Estrada, 2002).

El desconocimiento de la biología de la plaga y ausencia de un manejo integrado por parte de los productores, ha conllevado al uso de una amplia gama de insecticidas fosforados que ha generado problemas en los bovinos, producción de leche y medio ambiente (Morales \& Rodriguez, 2004; Vergara, 2006). Debido a esto se han creado iniciativas para el control de la chinche de los pastos por medio de enemigos naturales. Entre estos, se ha observado control depredador de Alpaida variabilis Keyserling (Aranae: Areaneidae) (Flórez et al., 2004) y Eriopis connexa connexa Germar (Coleoptera: Coccinelidae) (Martínez \& Barreto,1998). Pese a este control natural, se ha evidenciado que dichos depredadores se mantienen en densidades poblacionales mínimas respecto a la plaga y su consumo promedio diario de la chinche de los pastos es bajo (Flórez et al., 2004; Martínez \& Barreto, 1998). En cuanto a entomopatógenos se ha evaluado la patogenicidad en laboratorio del hongo Beauveria bassiana (Bals.) Vuill (Ascomycota: Clavicipitaceae), sin embargo no ha sido posible establecer si su aplicación tiene influencia en la disminución de las poblaciones de la chinche en campo (Vergara, 2006).

Los nematodos entomopatógenos (NE) de las familias Steinernematidae y Heterorhabditidae han mostrado ser altamente eficientes en el control de plagas en pastizales en Estados Unidos, Korea, Japón y Australia. Se destacan las especies Steinernema glaseri Steiner, S. kushidai Mamiya, S. scarabaei Stock \& Koppenhöfer, Heterorhabditis megidis, Poinar, Jackson \& Klein, H. bacteriophora Poinar, H. zealandica Poinar, H. marelata Liu \& Berry (Grewal et al., 2005). Los NE presentan alta virulencia, alto potencial reproductivo, pueden criarse de forma masiva en laboratorio, son inocuos para el ser humano y animales domésticos y tienen un amplio rango de acción (Sáenz, 2011). En evaluaciones de laboratorio con las especies Steinernema sp. cepa JCL024 y Heterorhabditis sp. cepa SL0708, se comprobó que estos nematodos ocasionan $75 \%$ hasta $100 \%$ de mortalidad en adultos y ninfas de la chinche de los pastos entre las 24 y 48 horas (Naranjo et al., 2012). Por ende, el objetivo de esta investigación fue evaluar la eficacia de Steinernema sp. JCL024 y Heterorhabditis sp. SL0708 en la chinche de los pastos C. scenica bajo condiciones de invernadero y establecer el número de JI recuperados de los cadáveres después de la aplicación.

\section{Materiales y Métodos}

\section{Obtención de nematodos e insectos}

Los NE Steinernema sp. JCL024, aislados del municipio Buenavista-Quindío, Colombia (acuerdo 182 CenicaféPontificia Universidad Javeriana PUJ) y Heterorhabditis sp. SL0708, aislados del municipio Alcalá, Valle del Cauca, Colombia, PUJ, se multiplicaron en larvas de Galleria mellonella (L) (Lepidoptera: Pyralidae), siguiendo la metodología de Sáenz \& López (2011). Para los bioensayos se aplicaron juveniles infectivos (JI) de 8 días de almacenamiento.

Para la cría de la chinche, se colectaron adultos en pastizales de kikuyo en el municipio de Guachetá (Cundinamarca, Colombia) $05^{\circ} 23^{\prime} 11^{\prime \prime} \mathrm{N}-73^{\circ} 41^{\prime} 24$. Estos se ubicaron en macetas de $1.5 \mathrm{~L}$ con pasto kikuyo dentro de casas de malla de $1 \times 1 \times 1 \mathrm{~m}$ a $19{ }^{\circ} \mathrm{C} \pm 3$ en el día y $8-12{ }^{\circ} \mathrm{C} \pm 2$ en la nochey $75 \%$ humedad relativa.

\section{Bioensayo en invernadero}

En unidades experimentales (UE), conformadas por recipientes plásticos de $50 \mathrm{~cm} 3$ y pasto kikuyo en estado vegetativo ( $7 \mathrm{~cm}$ de alto y 33 hojas), se ubicaron 5 adultos y 5 ninfas de C. scenica, siguiendo las recomendaciones de Martínez \& Barreto (1998). Cada UE se cubrió con bolsas plásticas trasparentes para evitar el escape de las chinches. Cada 3 días se aplicaron $10 \mathrm{ml}$ de agua para garantizar la humedad del substrato en las UE. Con un aspersor manual, se aplicaron los JI en agua destilada y Tween 20 al $0.5 \%$. Se evaluaron 5 dosis de JI/ $\mathrm{cm}^{2}$ para cada NE (Tabla 1), de acuerdo a los resultados obtenidos por Naranjo et al., (2012) en laboratorio. Cada tratamiento contó con 5 repeticiones. Durante todo el ensayo las UE se mantuvieron en invernadero

Tabla 1. Dosis evaluadas de Heterorhabditis sp. SL0708 y Steinerrnema sp. JCL024 sobre Collaria scenica en condiciones de invernadero

\begin{tabular}{|c|c|}
\hline Especie & Dosis JI/cm² foliolo \\
\hline \multirow{4}{*}{ Heterorhabditis sp. SL0708 } & 2 \\
\hline & 16 \\
\hline & 78 \\
\hline & 160 \\
\hline \multirow{4}{*}{ Steinerrnema sp. JCL024 } & 2 \\
\hline & 16 \\
\hline & 78 \\
\hline & 160 \\
\hline Sin nematodos & 0 \\
\hline
\end{tabular}

Jl: juveniles infectivos 
bajo las mismas condiciones descritas anteriormente. Se evaluó por 21 días cada 24 horas, realizando el conteo de chinches muertas y valoración del daño del pasto siguiendo la escala de evaluación propuesta por Martínez y Barreto (1998) (Tabla 2). Los insectos muertos fueron llevados a cámaras White (White, 1927) para el conteo de JI producidos hasta el agotamiento del cadáver.

Tabla 2. Escala visual de daño en pasto kikuyo (Pennisetum clandestinum) por Collaria scenica

\begin{tabular}{cll}
\hline Nivel & \multicolumn{1}{c}{ Daño } & \multicolumn{1}{c}{ Descripción } \\
\hline 0 & Sin daño & Pasto sano \\
1 & Leve & Presencia de puntos blancos \\
2 & Moderado & Amarillamiento bordes y ápice \\
3 & Grave & Necrósis apical y enrollamiento. \\
\hline
\end{tabular}

\section{Análisis estadístico.}

Los datos fueron analizados por análisis de varianza (ANOVA) y las medias comparadas por una prueba de Tukey $(\mathrm{P}<0.05)$, con ayuda del software Stadistics 17.0.

\section{Resultados y Discusión}

Se obtuvo un porcentaje de mortalidad del $95 \%$ en las ninfas de la chinche de los pastos con Steinernema sp. JCL024 y 88\% con Heterorhabditis sp. SL0708, 24 horas después de la infección (DDI). A partir del tercer día los tratamientos con Steinernema sp. JCL024 alcanzaron el 40\% en ninfas y adultos, excepto para la dosis de $16 \mathrm{JI} / \mathrm{cm}^{2}$ que presentó $37 \%$ y al noveno día, se destacaron las dosis de 78 y $160 \mathrm{JI} / \mathrm{cm}^{2}$ con $88 \%$ y $92 \%$ respectivamente, mientras para Heterorhabditis sp. SL0708 se registró $78 \%$ con16 JI/ $\mathrm{cm}^{2}$ (Figura 1). Porcentajes de mortalidad superiores al
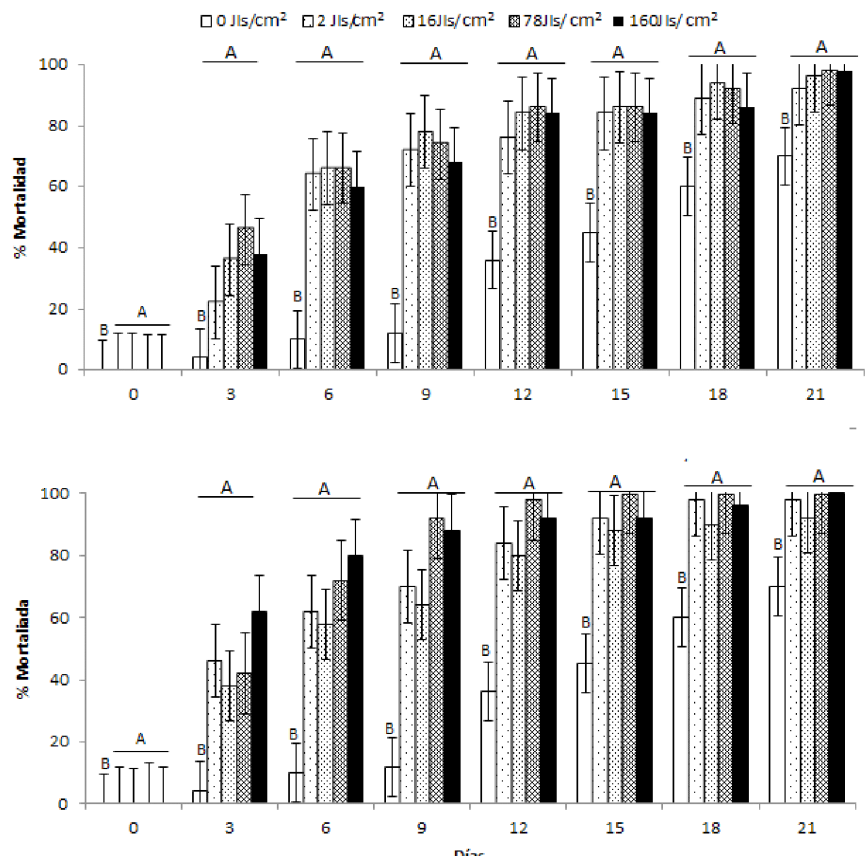

Figura 1. Porcentaje de mortalidad en individuos de Collaria scenica con nematodos entomopatógenos. A. Heterorhabditis sp. SL0708. B. Steinerrnema sp. JCL024. Las letras encima de las barras indican que no existen diferencias significativas entre las dosis $(P<0.05$, Tukey's). JIs: juveniles infectivos
$80 \%$ en los tratamientos con Heterorhabditis sp. SL0708 se alcanzaron sólo hasta el doceavo día ( $84 \%, 86 \%$ y $84 \%$ en las dosis 16,78 y $160 \mathrm{JI} / \mathrm{cm}^{2}$, respectivamente). A los 21 DDI, todos los tratamientos con NE superaron el 90\% (Figura 1). Sin embargo, no se presentaron diferencias significativas entre las dosis evaluadas $(F=0,058 ; d f=4,390 ; P=0,994)$ y las especies de nematodos aplicadas a la chinche de los pastos $(\mathrm{F}=0,014 ; \mathrm{df}=1,390 ; \mathrm{P}=0,906)$. No obstante, se observó que Steinernema sp. JCL024 produjo mayor mortalidad en menor tiempo comparado con Heterorhabditis sp. SL0708. El porcentaje de mortalidad en el tratamiento sin nematodos a los 9 DDI no superó el 12\%, sin embargo al final del ensayo llego al 70\% (Figura1A y 1B).

Batista \& Machado (2010), reportaron resultados similares de mortalidad a los obtenidos con la chinche de los pastos, quienes no encontraron diferencias significativas al evaluar 2.000 y $4.000 \mathrm{JIs} / \mathrm{ml}$ de Steinernema carpocapsae Weiser, $S$. feltiae Filipjev, S. riobravis Cabanillas, Poinar, \& Raulston y Heterorhabditis amazonensis RSC1 Andaló, Nguyen, \& Moino, en ninfas de Mahanarva spectabilis Distant (Hemíptera: Cercopidae). Marquez et al. (2005) encontraron que cepas aisladas del género Heterorhabditis mostraron mortalidad significativa con 3.000 y 6.000 JI en la chinche hedionda Scaptocoris talpa Champion, (Hemiptera: Cydnidae). Naranjo et al. (2012) bajo condiciones de laboratorio evaluando Steinernema sp. JCL024 y Heterorhabditis sp. SL0708 registraron un porcentaje de mortalidad en la chinche de los pastos y daño en las hojas del pasto kikuyo por C. scenica similar a lo observado en invernadero. Sin embargo, en invernadero, la mortalidad en ninfas y adultos de la chinche de los pastos fue mayor para Steinernema sp. JCL024 comparado con Heterorhabditis sp. SL0708, posiblemente debido a su patogenicidad y a la capacidad de búsqueda de los juveniles infectivos. En cuanto a la mortalidad que registró el tratamiento sin nematodos al final del ensayo, se relaciono con el nivel de daño tres presentado en las hojas, según Martínez y Barreto, (1998) el ataque de la chinche en este nivel es caracterizado por la muerte de los tejidos y ausencia de recurso alimenticio suficiente para mantener a todos los individuos en cada UE. En contraste, los tratamientos inoculados con Steinernema sp. JCL024 y Heterorhabditis sp. SL0708, no alcanzó el nivel 2 de daño y $100 \%$ de mortalidad de los insectos.

En cuanto a daño, el tratamiento sin nematodos mostró diferencias significativas $(\mathrm{F}=13,85 ; \mathrm{gl}=4,390 ; \mathrm{P}<0,0001)$, alcanzando necrosis y enrollamiento apical en las hojas, mientras las plantas tratadas con nematodos presentaron daño leve hasta los $21 \mathrm{DDI}(\mathrm{F}=0,225 ; \mathrm{gl}=1,390 ; \mathrm{P}=0,635)$, (Figura 2). El presente estudio proporcionó evidencia de que Steinernema sp. JCL024 y Heterorhabditis sp SL0708, ejercieron control y mantuvieron bajo el daño hasta por 21 días. Rangos similares de mortalidad se han logrado contra minadores de hoja, con modificación en los rangos y condiciones de aplicación (Williams \& Walters, 2000). No obstante, se debe seguir desarrollando estudios para optimizar la aplicación de Steinernema sp. JCL024 y Heterorhabditis sp. SL0708 en condiciones de campo para el buen control de la chinche de los pastos. 

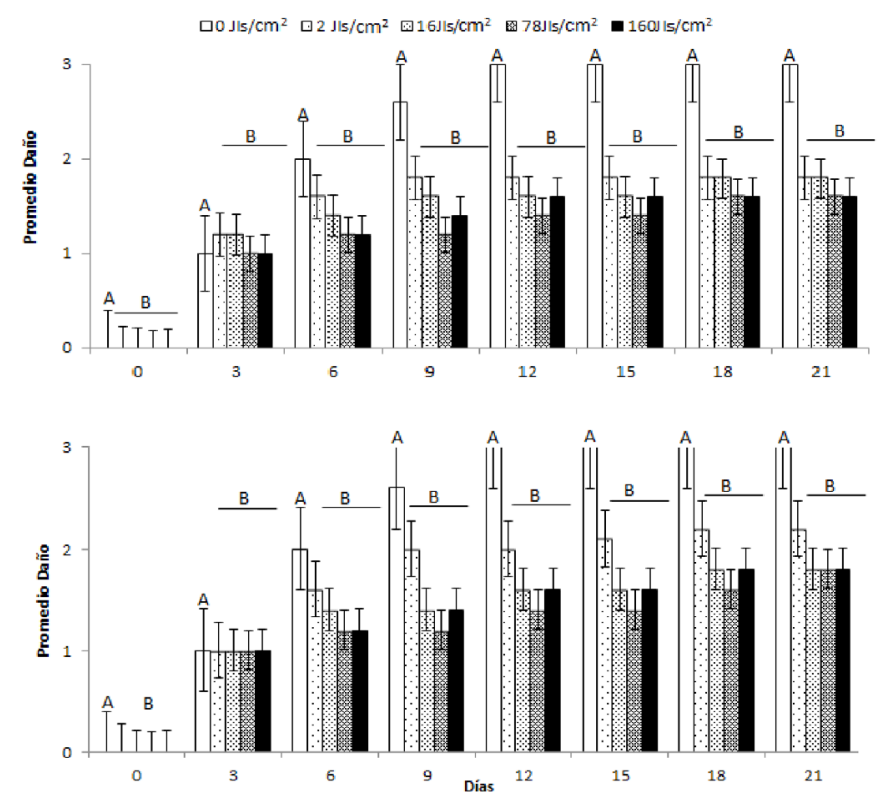

Figura 2. Promedio de daño en hojas de Pennisetum clandestinum por Collaria scenica inoculados con cuatro dosis de nematodos entomopatógenos. A. Heterorhabditis sp.SL0708. B. Steinerrnema sp. JCL024. Las letras encima de las barras indican que no existen diferencias significativas entre las dosis $(P<0.05$, Tukey's). Jl: juveniles infectivos.

Al no existir trabajos en invernadero y campo con la chinche de los pastos y NE, la aplicación en césped para plagas del orden Hemíptera, ha sido principalmente para el control de Balanococcus takahashii McKenzie (Coccidae), Aspidiella phragmis (Ckll)(Diaspididae), Margarodes sp.(Margarodidae), las cuales causan serios problemas en los campos de golf en Estados Unidos (Watschke et al., 1995) y Korea (Choo et al., 2000). Head et al. (2007), Cuthbertson et al. (2007), Leite et al. (2005) y Martínez (2011), realizaron estudios en invernadero y campo con especies de NE de los géneros Steinernema y Heterorhabditis sobre otras especies de Hemípteros como Bemisia tabaci Genn (Aleyrodidae), Mahanarva fimbriolata Fabr. (Cercopidae) y Dictyla monotropidia Stal (Tingidae) respectivamente. Estos estudios reportaron 36\% hasta $\geq 90 \%$ de mortalidad en los primeros 15 días después de la infección.

Se observó cambio de coloración en los insectos inoculados. En estado normal, la chinche es marrón claro en el tórax y verde en el abdomen debido a la ingesta del tejido vegetal. Los individuos tratados con Heterorhabditis sp. SL0708 exhibieron coloración rojiza, mientras que los tratados con Steinernema sp. JCL024, marrón oscura. Además, la producción de juveniles infectivos por la chinche, se inició a partir del sexto DDI y se recuperaron en promedio $13.000 \mathrm{JI} / \mathrm{ml}$ para ambas especies (Figura 3). No existen diferencias significativas en relación a los JI recuperados entre las especies de $\mathrm{NE}(\mathrm{F}=0,204 ; \mathrm{gl}=3,7$; $\mathrm{P}=0,893)$ y las dosis aplicadas $(\mathrm{F}=0,490 ; \mathrm{gl}=1,7 ; \mathrm{P}=0,487)$. Sin embargo, Steinernema sp. JCL024 tiene mayor recuperación de JI en las dosis de 78 y $160 \mathrm{JI} / \mathrm{cm}^{2}$.

La relación positiva entre la patogenicidad de los JI y el número de juveniles de la progenie, se reflejó en el alto número de JI que penetraron la chinche de los pastos, lo cual se atribuyó a la reproducción dentro del insecto y al tamaño del nematodo, ya que individuos grandes como es el caso de $S$. glaseri, ocupan más espacio y como consecuencia el

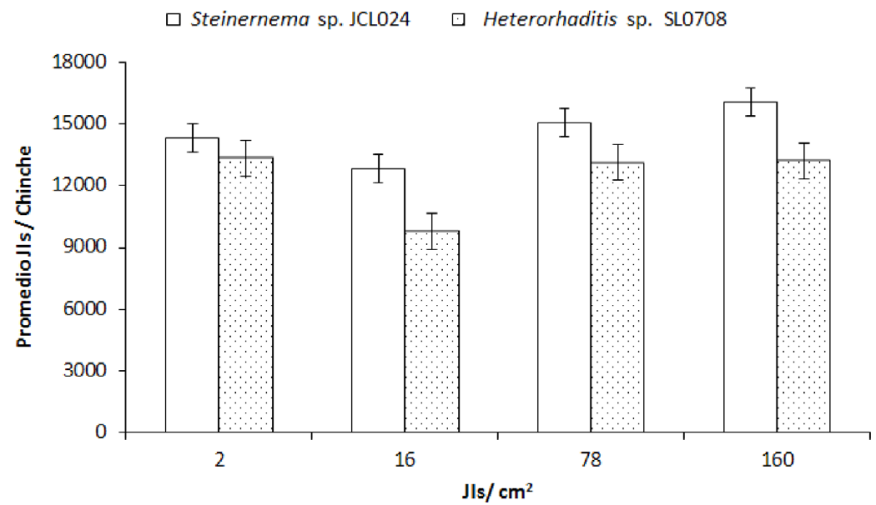

Figura 3. Producción de juveniles infectivos (JI) en chinche de los pastos A. Heterorhabditis sp.SL0708. B. Steinerrnema sp. JCL024

número de individuos recuperados de un hospedero es bajo, a comparación de Steinernema sp. JCL024 y Heterorhabditis sp. SL0708, que son individuos más pequeños (LópezNúñez et al., 2007; Sáenz \& López, 2011). Por otra parte, el cambio de coloración fue más evidente en el abdomen y en el tórax de ninfas y adultos de la chinche, lo cual concuerda con los resultados obtenidos en laboratorio por Naranjo et al. (2012).

\section{Conclusiones}

Los NE Steinernema sp. JCL024 y Heterorhabditis sp. SL0708 mostraron alta eficacia sobre la mortalidad de ninfas y adultos de $C$. scenica en condiciones de invernadero. Además de la reducción del daño ocasionado por la chinche, especialmente con las dosis de 78 y $160 \mathrm{JI} / \mathrm{cm}^{2}$. Es por ello que se hace necesario complementar estudios en el cual se combine el uso de estos dos nematodos y a mediano plazo, se puedan integrar dentro de programas de manejo en las praderas ganaderas.

\section{Literatura Citada}

Barboza, M. R. Collaria scenica (Stal, 1859) (Hemíptera: miridae) em poaceas hibernais na região centro sul do Paraná: biologia e danos. Universidade Estadual do CentroOeste, Unicentro-PR. 2009.67p. Tesis Maestría.

Batista, E.S.P; Machado, A. Application methods of entomopathogenic nematodes for control of Mahanarva spectabilis (Hemíptera: Cercopidae). Biocontrol Science and Technology,20: 1079-1085, 2010. <http://dx.doi.org/1 0.1080/09583157.2010.515300>. 17 Jun. 2012.

Briceño, A. J. Otra chinche más en el pasto kikuyo del estado de Mérida. Boletín Divulgativo del Instituto de Investigaciones Agropecuarias, v.30, n.60, p.31-32, 2007. $<$ http://www.saber.ula.ve/bitstream/123456789/18582/2/ articulo8.pdf>. 17 Jun. 2012.

Carlessi, L.R.G.; Corseuil, E.; Salvadori, J.R. Aspectos biológicos e morfométricos de Collaria scenica (Stal) (Hemiptera: Miridae) em trigo. Anais da Sociedade Entomológica do Brasil, v.28, n.1, p.65-73, 2001. <http:// dx.doi.org/10.1590/S0301-80591999000100007> 
Choo, H.Y.; Lee, D.W.; Lee, S.M.; Lee, T.W.; Choi, W.G., Chung, Y.K.; Sung, Y.T. Turfgrass insect pests and natural enemies in golf courses. Korean Journal of Applied Entomology, v.39, n. 3, p.171-179, 2000. <http://www.cabi.org/isc/Default.asp $\mathrm{x}$ ? site $=144 \&$ page $=2540 \&$ LoadModule $11=\mathrm{CABISEARCH}$ RESULTS\&LoadAction $=$ LoadAbstract\&term $=\mathrm{au} \% 3 \mathrm{~A} \% 22$ Lee+DongWoon $\% 22 \&$ AbstractSearchTerm $=a \mathrm{au} \% 3 \mathrm{~A} \% 22 \mathrm{Le}$ $\mathrm{e}+$ DongWoon $\% 22 \&$ query $=\mathrm{au} \% 3 \mathrm{~A} \% 22 \mathrm{Lee}+$ DongWoon $\% 2$ 2\&AbstractID=20013016556>. 12 Sep. 2012.

Cuthbertson, A.G.S.; Walters, K.F.A.; Northing, P.; Luo, W. Efficacy of the entomopathogenic nematode, Steinernemafeltiae, against sweet potato white fly Bemisiatabaci (Homoptera: Aleyrodidae) under laboratory and glasshouse conditions. Bulletin of Entomological Research, v. 97, n.1, p.9-14, $2007<$ <ttp://dx.doi. org/10.1017/S0007485307004701>

Estrada, J. Pastos y forrajes para el trópico colombiano. Manizales: Editorial Universidad de Caldas, 2002. 511p.

Ferreira, E.; Barrigossi, J.A.F.; Vieira, N.R. Percevejos das panículas do arroz: fauna Heteroptera associada ao arroz. Santo Antônio de Goiás: Embrapa Arroz Feijão, 2001. 52p. (Embrapa Arroz e Feijão. Circular Técnica, 43).

Flórez, E.; Pinzón, J.; Sabogal, A.; Barreto, N. Selección de presas y composición de la dieta de la arañaAlpaidavariabilis (Araneae: Araneidae) en pastizales de la sabana de Bogotá, Colombia. Revista Ibérica de Aracnología, v.9, n.1, p.241-248, 2004. <http://www.sea-entomologia.org/ Publicaciones/RevistaIbericaAracnologia/RIA09/R09028-241.pdf>. 30 Jun. 2012.

Grewal, P.S.; Koppenhöfer, A.M.; Choo, H.Y. Lawn, turfgrass and pasture applications. P 115-141. In: Grewal P.S.; Ehlers R.U.; Shapiro-Ilan, D.I. (Eds.). Nematodes as biocontrol agents. Wallingford: CAB International, 2005. p. 115-146.

Head, J.; Lawrence, A.J.; Walters, A.J. Efficacy of the entomopathogenic nematode, Steinerrnema feltiae, against Bemisiatabaci in relation to plant species. Journal of Applied Entomology, v. 128, n.8, p.543-547, 2007. <http:// dx.doi.org/10.1017/S0007485307004701>

Leite, L.G.; Machado, L.A.; Goulart, R.M.; Tavares F.M.; Batista Filho, A. Screening of entomopathogenic nematodes (Nemata: Rhabditida) and the efficiency of Heterorhabditis sp. against the Sugarcane Root Spittlebug Mahanarva fimbriolata (Fabr.) (Hemiptera: Cercopidae). Neotropical Entomology, v.34, n.5, p.785-790, 2005. <http://dx.doi. org/10.1590/S1519-566X2005000500010>

López-Núñez, J.C.; Cano, L.; Gongora-B, C.; Stock, P. Diversity and evolutionary relationships of entomopathogenic nematodes (Steinernematidae and Heterorhabditidae) from the Central Andean region of Colombia. Nematology, v.9, n.3, p.333-341, 2007. <http:// dx.doi.org/10.1163/156854107781351972>

Marquez, J.M.; Ralda, G.; López, E.; Maldonado, D. Parasitismo de dos nematodos entomopatógenos (Heterorhabditis spp.) sobre Chinche hedionda (Scaptocoris talpa, Hemiptera: Cydnidae) y Gusano alambre (Dipropus spp. Coleoptera: Elateridae), bajo condiciones de laboratorio. In: Cengicaña (Ed.). Memoria: presentación de resultado de investigación Zafra 2004-2005. Guatemala: Cengicaña, 2005. p.
89-95. $\quad<$ http://www.cengicana.org/es/publicaciones/ memorias/memorias/2004-2005/manejo-integrado-plagas/ Parasitismo-de-dos-nematodos-entomopat $\% \mathrm{C} 3 \% \mathrm{~B} 3$ genos(Heterorhabditis-spp)-sobre-Chinche-hedionda(Scaptocoris-talpa-HemipteraCydnidae)-y-Gusanoalambre-(Dipropus-spp.-ColeopteraElateridae)-bajocondiciones-de-laboratorio/>. 07 Oct. 2012.

Martinez, G. E; Barreto, N. La chinche de los pastos Collaria scenica Stal en la Sabana de Bogotá. Santafé de Bogotá: Corpoica, 1998. 66p.

Martínez, H. E. Aspectos de la biología de la chinche de encaje Dictyla monotropidia Stal. (Hemiptera: Tingidae) y evaluación de la eficacia de entomopatógenos. Universidad Nacional de Colombia. Sede Medellín, 2011, 84p. Tesis Maestría.

Melo, M.C.; Dellapé, P.M.; Carpintero, D.L.; Coscarón, M.C. Reduviidae, Miridae y Lygaeoidea(Hemíptera) recolectados en Colonia Carlos Pellegrini (Esteros de Iberá, Corrientes, Argentina). Revista da Sociedad Entomológica Argentina, v.63, n.1-2, p.59-67, 2004.<http://www.scielo.org.ar/scielo. php?script $=$ sci_arttext\&pid $=$ S0373-56802004000100012> 12 Ene. 2012.

Morales, C.A.; Rodriguez, N. El Cloropirifos: posible disruptor Endócrino en bovinos de leche. Revista Colombiana de Ciencias Pecuarias, v.17, n.3, p.255- 267, 2004. <http:// rccp.udea.edu.co/index.php/ojs/article/viewFile/174/172> 22 Dic. 2011.

Naranjo, N.; Villamil, D.A.V; Sáenz, A. Primer reporte de patogenicidad por nematodos entomopatogenos sobre la chinche de los pastos Stal (Hemiptera: Miridae). Entomotropica, v.26, n.3, p 117-125, 2011. <http:// entomotropica.org/index.php/entomotropica/article/ viewFile/318/360>. 8 Mar. 2012.

Sáenz, A. Heterorhabditis sp. SL0708 como una alternativa para el control de plagas. In: Sáenz. A.; López. J.C; Galindo, L. (Eds). Experiencias en nematodos entomopatógenos: retos y oportunidades de uso en Latinoamérica. Bogotá: COHIBO-Pontificia Universidad Javeriana, 2011. p.2-12.

Sáenz, A.; López, J.C. Ciclo de vida y patogenicidad del aislamiento nativo Heterorhabditis sp. SL0708 (Rhabditida: Heterorhabditidae). Revista Colombiana de Entomología, v.37, n.1, p,43-47, 2011. <http://www. scielo.org.co/scielo.php?script $=$ sci_arttext\&pid $=\mathrm{S} 0120$ 04882011000100007\&lng=pt\&nrm=iso>. 22 Jun. 2011.

Vergara, R.R. Collaria spp. Insecto Dañino del kikuyo: Métodos de Control. In: Seminario internacional competitividad en carne y leche, 5., 2006, Medellín. Anais. Medellín: COLANTA, 2006. p. 197-231.

Watschke, T.L.; Dernoeden, P.H.; Shetlar, D.J. Managing turfgrass pests. Boca Raton, Florida: Lewis Publishers, 1995. p.171-343.

White, G.F. A method for obtaining infective nematodes larvae from cultures.Science, v.66, n.1709, p.302-303, 1927. <http:// dx.doi.org/10.1126/science.66.1709.302-a>

Williams, E.C. ; Walters, K.F.A. Foliar application of the entomopathogenic nematode, Steinernema feltiae against leafminers on vegetables. Biocontrol Science and Technology, v.10, n.1, p.61-70, 2000. <http://dx.doi. org/10.1080/09583150029396> 